\title{
Recherche de géniteurs de résistance à la fusariose de l'épi causée par Fusarium culmorum chez le blé et les espèces voisines
}

\author{
L Saur \\ avec la collaboration technique de JY Morlais \\ INRA, station d'amélioration des plantes, domaine de la Motte-au-Vicomte, BP 29, 35650 Le Rheu, France
}

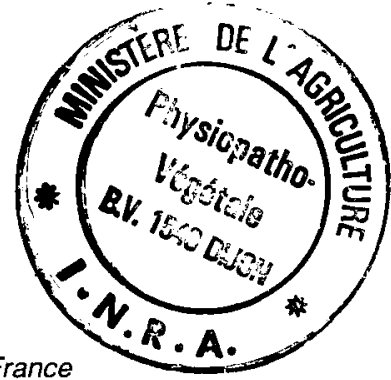

(Reçu le 21 décembre 1990; accepté le 29 avril 1991)

\begin{abstract}
Résumé - Sur une période de 10 ans, 230 variétés de blé tendre et 334 lignées appartenant à 15 espèces de triticinées ont été évaluées pendant une ou plusieurs années pour leur résistance à la fusariose de l'épi causée par Fusarium culmorum. La contamination a été réalisée par pulvérisation d'une suspension de spores à l'anthèse pour chaque génotype. Aucun niveau de résistance très élevé n'a été trouvé chez les espèces voisines de Triticum aestivum. Les lignées de blé de printemps des zones subtropicales signalées pour leur résistance à Fusarium graminearum se sont montrées assez résistantes à $F$ culmorum dans nos conditions. Les blés tendres d'hiver étudiés présentent une variabilité importante pour la résistance avec de très grandes différences entre les variétés peu sensibles et les variétés très sensibles. Le niveau de résistance à rechercher pour des variétés de blés d'hiver en zone tempérée est discuté à partir de mesures de l'effet du parasite sur le rendement.
\end{abstract}

\section{Fusarium culmorum / résistance / blé / Triticeae}

Summary - Sources of resistance to head blight caused by Fusarium culmorum in bread wheat and related species. Over a 10-yr period, a total of 230 wheat genotypes and 334 lines from 15 Triticineae species were evaluated for their resistance to head blight caused by Fusarium culmorum. Plants were inoculated in the field by spraying a spore suspension at the anthesis of each genotype. The percentage of infected spikelets was evaluated visually $20 \mathrm{~d}$ after contamination and the effect of the disease on thousand grain weight was measured by comparing inoculated and uninoculated plots. The same 4 varieties were used as control every year. The good difference between the susceptible and the resistant controls (fig 1), provided a scale to compare genotypes even if they were not tested during the same years. A wide genetic variation was found in winter wheat, from very susceptible to partially resistant genotypes (table 1). The resistance level needed for winter wheat cultivation in the temperate climate of northern France is discussed according to the evaluation of the loss of grain yield due to the disease (table II). Exotic spring wheat varieties reported for their tolerance to Fusarium graminearum were also tolerant to $\mathrm{F}$ culmorum under our environmental conditions (table III). Related species did not display a very high level of resistance (table IV). Only the frequency of resistant genotypes is higher in the species Triticum monococcum and Aegilops speltoides.

Fusarium culmorum / resistance / wheat / Triticeae

\section{INTRODUCTION}

La fusariose de l'épi provoque chez le blé des pertes de rendement qui peuvent être très importantes en cas d'attaque grave, mais aussi une contamination du grain par des mycotoxines qui peuvent déprécier la qualité de la récolte. II n'existe pas pour l'instant de possibilité de lutte chimique efficace contre cette maladie fongique qui se développe lorsqu'une période humide se produit au moment de la floraison. Des différences de comportement entre génotypes de blé tendre ont été signalées dès 1942 par Dickson, mais aucun niveau de résistance très élevé n'a été trouvé. Les résultats les plus nombreux concernent les blés de printemps cultivés dans les régions à climat chaud et humide où l'agent responsable de la maladie est Fusarium graminearum (Schwabe). Dans les conditions plus fraîches de l'Europe du Nord-Ouest, le dévelop- 
pement des épidémies est moins fréquent et est dû surtout à Fusarium culmorum (WG Smith). Jusqu'à présent, aucune interaction entre des génotypes de blé et ces 2 espèces fusariennes n'a été trouvée. Mesterhazy (1988) évalue à 0,90 le coefficient de corrélation entre les résultats obtenus sur plusieurs génotypes pour ces 2 espèces en contamination artificielle.

Les données concernant la variabilité de la résistance à $F$ culmorum chez le blé sont peu nombreuses (Walther, 1976; Mielke, 1988; Snijders, 1990). La variation des conditions climatiques durant les mois de mai et juin rend difficile la comparaison de variétés de précocités différentes. La relation entre les symptômes à un stade donné et la perte de rendement causée par le parasite dépend de la variété (Saur et Benacef, 1991). Une bonne connaissance du comportement d'un génotype nécessite une expérimentation pluriannuelle et la prise en compte de l'effet de la maladie sur le rendement.

Dans cette étude nous présentons les résultats obtenus sur 10 années dans des essais destinés à identifier des sources de résistance à la fusariose de l'épi causée par Fusarium culmorum utilisables pour l'amélioration du blé tendre d'hiver en climat tempéré.

\section{MATÉRIEL ET MÉTHODES}

\section{Matériel végétal}

Les essais ont été réalisés entre 1981 et 1990 sur 3 types de matériel végétal : des blés de printemps originaires de différentes parties du monde où la fusariose de l'épi est répandue, des blés d'hiver européens et une collection de quelques espèces de triticinées. Cent quarante sept variétés de blé de printemps ont été étudiées. Certaines ont été introduites à partir des données bibliographiques et tous les blés originaires de Chine présents dans notre collection ont été testés. Les 83 génotypes de blé d'hiver expérimentés représentent un échantillon de variétés françaises signalées pour leur bon comportement vis-àvis de la fusariose de l'épi ou largement cultivées ou nouvelles.

Des lignées d'Europe du Sud provenant d'échanges avec l'institut de recherche sur les céréales (GKI) en Hongrie, l'université de Belgrade en Yougoslavie et l'institut de recherche sur les céréales de Fundulea en Roumanie ont été également mises en essai. Pour les espèces voisines du blé tendre, les 334 lignées étudiées proviennent de la collection de la station d'amélioration des plantes de Rennes et représentent 15 espèces.

\section{Protocole expérimental}

Tous les tests ont été réalisés au champ en semis d'automne avec contamination artificielle par pulvérisation de spores sur les épis. L'inoculum est constitué d'une suspension de conidies dans de l"eau (106 spores $/ \mathrm{ml}$ ). L'isolat de $F$ culmorum utilisé a été isolé à partir d'un épi infecté en conditions naturelles provenant de la région de Rennes. La contamination est réalisée le soir après une irrigation par aspersion afin de maintenir une humidité suffisante sur les plantes pendant la nuit.

Pour les blés tendres, le dispositif est le type crisscross avec 3 répétitions et 2 traitements séparés par une parcelle de bordure (traitement avec contamination et témoin sans contamination). La parcelle élémentaire est un poquet de 20 grains avec un maillage de $50 \mathrm{~cm}$ entre poquets. Chaque variété est contaminée individuellement au stade floraison.

Pour les espèces voisines, le premier tri a été fait avec 2 répétitions seulement et 3 contaminations de l'ensemble de l'essai depuis la floraison du génotype le plus précoce, jusqu'à celle du plus tardif. Les lignées les plus interessantes ont subi une deuxième ou une troisième année d'essai avec contamination individualisée à la floraison et 3 répétitions.

L'effet du parasite sur le rendement de quelques variétés a été mesuré dans un dispositif en parcelles plus grandes $\left(6 \mathrm{~m}^{2}\right)$ sur 3 années. En 1985, 2 concentrations d'inoculum $\left(10^{6}\right.$ et $2 \times 10^{5}$ spores $\left./ \mathrm{ml}\right)$ ont été utilisées pour ce type d'essai de façon à obtenir 2 niveaux de contamination.

\section{Évaluation de la sensibilité}

Des traitements fongicides destinés à éviter le développement du piétin verse, des rouilles et de l'oïdium ont été réalisés sur l'ensemble des essais.

L'intensité de la maladie a été notée 20-25 j après la contamination pour chaque génotype. La proportion d'épillets présentant des symptômes est appréciée visuellement selon une échelle de 1 (pas de symptômes) à 9 (100\% des épillets atteints). Dans les essais en parcelles de $6 \mathrm{~m}^{2}$, la notation des symptômes a été faite par dénombrement des épillets malades.

Le rendement en grain n'a été mesuré que dans les essais réalisés à cet effet. La petite taille des parcelles dans les essais en poquets ne permet pas une bonne évaluation du rendement, mais le poids de 1000 grains peut être estimé avec précision. Le coefficient de corrélation entre la perte de rendement et la perte de poids de 1000 grains causée par le parasite est élevé (Saur et Benacef, 1991). L'effet de la maladie a été étudié pour les blés d'hiver seulement, par le rapport du poids de 1000 grains de la parcelle contaminée sur le poids de 1000 grains de la parcelle non contaminée. Quatre variétés témoins représentant 2 groupes de précocité et de sensibilité différents ont été reconduites pendant les 10 années d'expérimenta- 
tion. II s'agit de Copain et Réso (précoces) et Bizel et Capest (tardifs).

Tous les génotypes n'ont pas été mis en essai les mêmes années. Pour pouvoir comparer les variétés expérimentées sur des périodes différentes, les résultats de chaque année ont été transformés à partir des valeurs obtenues pour le témoin résistant Bizel et le témoin sensible Réso. La transformation de type linéaire tient compte de la moyenne de ces 2 témoins et de leur écart l'année considérée et les résultats sont exprimés dans l'échelle de référence formée par les valeurs moyennes des témoins sur 10 ans.

\section{RÉSULTATS}

\section{Développement de la maladie}

Le développement de la maladie a été satisfaisant pour toutes les années d'observation. Les résultats des témoins (fig 1) montrent que la différence entre témoins sensibles et peu sensibles se manifeste de façon nette chaque année pour les 2 caractères étudiés : symptômes et effet du parasite sur le poids de 1000 grains. On distingue dans tous les cas les 2 variétés sensibles Capest et Réso des 2 variétés résistantes Bizel et Copain. Seule l'amplitude de la différence varie avec l'année.

\section{Évaluation des blés d'hiver}

Une grande variabilité de comportement a été trouvée dans les blés d'hiver. Le tableau I présente la moyenne des valeurs ajustées obtenues pour chaque variété pour les différentes années d'étude. Certains génotypes se montrent extrêmement sensibles : Rivoli ou Frandoc pour les types précoces ou Hobbit pour les tardifs. Leur sensibilité se rapproche de celle des variétés de blé dur expérimentées dans nos essais. Des variétés largement cultivées comme Festival, Fidel ou Arminda présentent un bon comportement. Ringo Sztar, variété hongroise ainsi que des lignées roumaines et yougoslaves se montrent assez résistantes. Elles sont issues de programmes de sélection incluant la résistance à la fusariose de l'épi dans leurs objectifs. Parmi les variétés ayant subi une seule année d'essai, Arina lignée suisse tardive a été peu attaquée par la parasite. Aucune variété n'a présenté un niveau de résistance meilleur que celui de Bizel.

L'effet de la maladie sur le rendement en grain de quelques variétés de sensibilités différentes
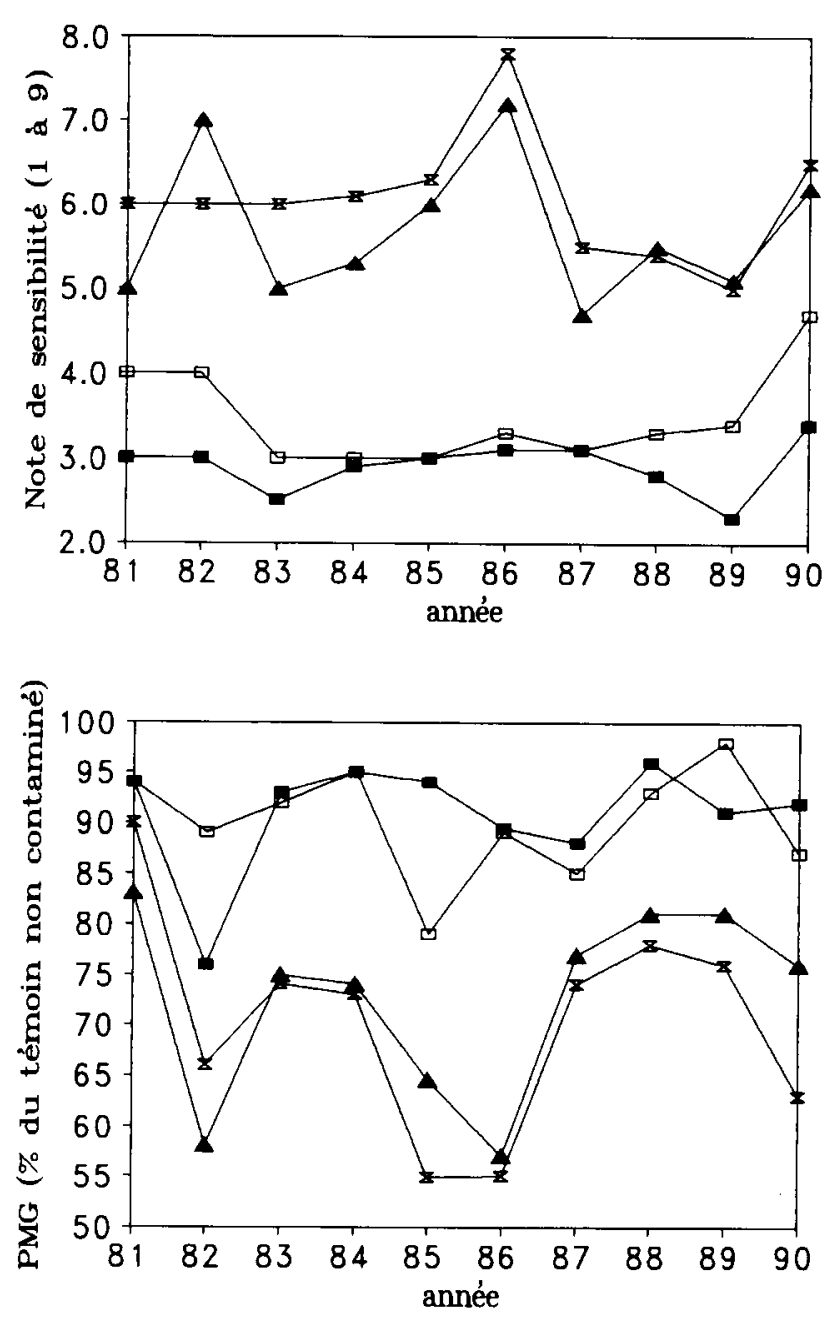

Fig 1. Note de sensibilité et poids de 1000 grains (PMG) dans les essais de résistance à la fusariose de l'épi causée par $F$ culmorum, pour 4 témoins sur 10 ans. $\longrightarrow$-Bizel; -A-Capest; - - - Copain; - $x$ - Reso.

est présenté dans le tableau II. L'importance de l'attaque est variable selon les essais. L'utilisation d'un inoculum de concentration plus faible en 1985 se traduit par un moindre développement de la maladie comparable à celui d'une année où les conditions climatiques ne sont pas favorables.

Trois types de situations peuvent être décrits à partir de ces résultats :

- en cas d'attaque très forte (1982) : la variété la plus résistante perd $23 \%$ de son rendement contre $62 \%$ pour la variété la plus sensible;

- en cas d'attaque moyenne comme en 1983 ou en 1985, le parasite a peu d'effet sur les variétés résistantes. Les témoins sensibles Réso ou Rescler subissent une baisse de rendement nette et des variétés extrêmement sensibles peuvent être fortement endommagées (Pernel et Frandoc, 1983); 
Tableau I. Comportement de variétés de blé d'hiver dans des essais de résistance à la fusariose de l'épi. Intensité de l'attaque et effet de la maladie sur le poids de 1000 grains.

\begin{tabular}{|c|c|c|c|}
\hline Nom & $\begin{array}{l}\text { Nombre } \\
\text { d'années } \\
\text { d'essai }\end{array}$ & Note $^{1}$ & $\begin{array}{l}\text { Poids de Pays } \\
1 \text { ooo grains d'origine } \\
\text { (en \% du témoin) } \\
\text { non contaminé }\end{array}$ \\
\hline
\end{tabular}

Variétés ayant subi plusieurs années d'essai

\begin{tabular}{|c|c|c|c|c|}
\hline Bizel2 & 10 & 2,9 & 90,9 & France \\
\hline Libellula & 2 & 2,9 & 89,7 & Italie \\
\hline $199 \mid 9-11$ & 2 & 2,9 & 89,0 & Roumanie \\
\hline Adam & 4 & 3,0 & 85,3 & France \\
\hline Festival & 5 & 3,0 & 82,5 & France \\
\hline Ringo Sztar & 3 & 3,1 & 86,5 & Hongrie \\
\hline $2325 W_{1-1}$ & 2 & 3,1 & 85,5 & Roumanie \\
\hline Ns 732 & 2 & 3,2 & 87,7 & Roumanie \\
\hline Renan & 5 & 3,2 & 86,0 & France \\
\hline $199 \mid 1-12$ & 2 & 3,2 & 77,1 & Roumanie \\
\hline Storch & 2 & 3,3 & 90,9 & Allemagne \\
\hline Turda 195 & 2 & 3,3 & 88,4 & Roumanie \\
\hline Transylvania & 2 & 3,3 & 88,0 & Roumanie \\
\hline $156 \mid 7-21$ & 2 & 3,3 & 85,6 & Roumanie \\
\hline Briscar & 2 & 3,3 & 80,0 & France \\
\hline $156 \mid 1-22$ & 2 & 3,4 & 82,1 & Roumanie \\
\hline $\mathrm{Zg} \mathrm{6103/84}$ & 2 & 3,4 & 77,5 & Yougoslavie \\
\hline Copain $^{2}$ & 10 & 3,5 & 90,1 & France \\
\hline $\mathrm{Zg} \mathrm{8029/82}$ & 3 & 3,5 & 87,4 & Yougoslavie \\
\hline Sabre & 4 & 3,5 & 83,6 & France \\
\hline lena & 5 & 3,5 & 76,6 & France \\
\hline $74-2$ & 3 & 3,6 & 92,8 & Hongrie \\
\hline E de Choisy & 3 & 3,6 & 87,3 & France \\
\hline Fleurus & 2 & 3,6 & 80,3 & France \\
\hline Talent & 5 & 3,6 & 80,3 & France \\
\hline Glanor & 2 & 3,6 & 78,1 & France \\
\hline Poncheau & 7 & 3,7 & 87,9 & France \\
\hline Arminda & 9 & 3,7 & 83,6 & Pays-Bas \\
\hline Fidel & 5 & 3,7 & 82,9 & France \\
\hline 199| 1-31 & 2 & 3,7 & 79,4 & Roumanie \\
\hline $81-28$ & 3 & 3,8 & 79,2 & Hongrie \\
\hline Fundulea 29 & 2 & 3,9 & 85,4 & Roumanie \\
\hline Apexal & 3 & 4,0 & 73,7 & France \\
\hline Albatros & 3 & 4,1 & 82,9 & France \\
\hline $81-50$ & 3 & 4,2 & 85,8 & Hongrie \\
\hline Zenith & 2 & 4,2 & 85,1 & Suisse \\
\hline Camp Remy & 6 & 4,2 & 79,4 & France \\
\hline Soissons & 2 & 4,2 & 72,7 & France \\
\hline Roazon & 2 & 4,2 & 72,5 & France \\
\hline Fortin & 2 & 4,3 & 85,9 & France \\
\hline Voyage & 2 & 4,3 & 80,2 & France \\
\hline $79-1 / 57$ & 3 & 4,5 & 76,9 & Hongrie \\
\hline Super Zlatna & 2 & 4,6 & 82,2 & Bulgarie \\
\hline Goelent & 3 & 4,6 & 74,4 & France \\
\hline Match & 2 & 4,6 & 71,6 & France \\
\hline Florin & 2 & 4,8 & 73,2 & France \\
\hline Gala & 3 & 4,8 & 71,7 & France \\
\hline
\end{tabular}

\begin{tabular}{|c|c|c|c|}
\hline Nom & $\begin{array}{l}\text { Nombre } \\
\text { d'années } \\
\text { d'essai }\end{array}$ & $\begin{array}{l}\text { Note }^{1} \\
\qquad \\
\qquad \\
n\end{array}$ & $\begin{array}{l}1 \text { Poids de Pays } \\
1 \text { ooo grains d'origine } \\
\text { (en \% du témoin) } \\
\text { non contaminé }\end{array}$ \\
\hline
\end{tabular}

\begin{tabular}{|c|c|c|c|c|}
\hline $80-1 / 70$ & 3 & 4,9 & 79,7 & Hongrie \\
\hline Divio & 3 & 4,9 & 69,1 & France \\
\hline Top & 2 & 5,0 & 80,6 & France \\
\hline Moulin & 3 & 5,2 & 72,6 & G Bretagne \\
\hline Pernel & 7 & 5,4 & 63,1 & France \\
\hline Rescler & 6 & 5,6 & 68,6 & France \\
\hline Capest $^{3}$ & 10 & 5,7 & 72,7 & France \\
\hline Thésée & 4 & 5,9 & 73,4 & France \\
\hline Apollo & 3 & 6,0 & 77,7 & Allemagne \\
\hline Réso 3 & 10 & 6,0 & 70,4 & Fance \\
\hline Récital & 2 & 6,1 & 74,4 & France \\
\hline Tarasque & 3 & 6,2 & 80,2 & France \\
\hline Rivoli & 6 & 6,2 & 66,5 & France \\
\hline Frandoc & 3 & 6,4 & 63,5 & France \\
\hline Hobbit & 3 & 6,5 & 74,8 & G Bretagne \\
\hline Beauchamp & 5 & 6,5 & 71,2 & France \\
\hline
\end{tabular}

Variétés ayant subi une seule année d'essai

\begin{tabular}{lllll} 
Arina & 1 & 2,5 & 90,0 & Suisse \\
Hardi & 1 & 3,1 & 90,9 & France \\
Nimbus & 1 & 3,8 & 81,0 & Allemagne \\
Open & 1 & 3,9 & 93,6 & France \\
Ecu & 1 & 4,2 & 82,4 & France \\
Genial & 1 & 4,2 & 81,0 & France \\
Fortal & 1 & 4,2 & 76,7 & France \\
Scipion & 1 & 4,3 & 83,5 & France \\
Lutin & 1 & 4,3 & 74,1 & France \\
Decibel & 1 & 4,4 & 84,5 & France \\
Artaban & 1 & 4,5 & 76,7 & France \\
Louvre & 1 & 4,5 & 76,7 & Pays-Bas \\
Courtot & 1 & 4,5 & 76,0 & France \\
Festin & 1 & 4,5 & 63,9 & France \\
Baroudeur & 1 & 4,9 & 82,4 & France \\
Capitole & 1 & 5,0 & 63,9 & France \\
Gavroche & 1 & 5,1 & 84,4 & France \\
Jade & 1 & 5,6 & 89,5 & France \\
Fandango & 1 & 5,6 & 69,0 & France \\
Delfi & 1 & 5,7 & 84,0 & France \\
Gerbier & 1 & 6,2 & 60,8 & France \\
Comtal & 1 & 6,4 & 68,5 & France \\
Fief & 1 & 6,5 & 73,9 & France \\
Ducat & 1 & 6,7 & 83,3 & France \\
Dolmen & 1 & 7,1 & 67,0 & France \\
\hline
\end{tabular}

1 : Echelle de 1 (pas de symptômes) à 9 (100\% des épillets atteints).

2 : Témoin résistant.

3 : Témoin sensible. 
Tableau II. Effet d'une attaque de Fusarium culmorum sur le rendement de variétés de blé tendre. Rendement de la parcelle contaminée en \% du témoin non contaminé et nombre d'épillets présentant des symptômes.

\begin{tabular}{|c|c|c|c|c|c|c|c|c|}
\hline & \multicolumn{2}{|c|}{1982} & \multicolumn{4}{|c|}{$\begin{array}{c}1983 \\
\text { Concentration de l'inoculum (spores } / \mathrm{ml})\end{array}$} & \multicolumn{2}{|c|}{1985} \\
\hline & \multicolumn{2}{|c|}{106} & \multicolumn{2}{|c|}{$10^{6}$} & \multicolumn{2}{|c|}{$10^{6}$} & \multicolumn{2}{|c|}{$2 \times 10^{5}$} \\
\hline & $R d t \% 1$ & $E F$ & $R d t \%$ & $E_{l}$ & $R d t \%$ & $E_{l}$ & $R d t \%$ & $E_{I}$ \\
\hline Bizel & $77,0^{a, 3}$ & 25 & $93,3^{a}$ & 6 & $96,9^{a}$ & 6 & $97,6^{a}$ & 2 \\
\hline Copain & $74,1^{a}$ & 29 & $101,3^{a}$ & 6 & & & & \\
\hline Adam & $71,6^{a}$ & 25 & $91,3^{a}$ & 25 & & & & \\
\hline Poncheau & $70,9 a$ & 21 & & & & & & \\
\hline $43-6$ & & & & & $93,9^{a b}$ & 8 & $95,4^{a}$ & 2 \\
\hline Fidel & & & & & $88,1 \mathrm{bc}$ & 7 & $97,6^{a}$ & 4 \\
\hline Talent & & & & & $80,9^{\text {cd }}$ & 18 & $95,9^{a}$ & 4 \\
\hline Arminda & & & & & $74,5^{d}$ & 27 & $84,5^{b}$ & 16 \\
\hline Tarasque & & & $80,2^{\mathrm{bc}}$ & 47 & & & & \\
\hline $108-2$ & & & $75,6^{c}$ & 46 & & & & \\
\hline Rescler & $41,1^{b}$ & 95 & $74,8^{\mathrm{c}}$ & 42 & $53,6^{e}$ & 46 & $73,0^{c}$ & 30 \\
\hline Réso & $37,8^{b}$ & 75 & $75,5^{\mathrm{c}}$ & 39 & & & & \\
\hline Match & & & $74,1^{\mathrm{c}}$ & 39 & & & & \\
\hline Divio & & & $72,4^{c}$ & 37 & & & & \\
\hline Pernel & & & $57,1^{d}$ & 57 & & & & \\
\hline Frandoc & & & $50,3^{d}$ & 90 & & & & \\
\hline
\end{tabular}

$1:$ Rdt\% : Rendement après contamination en \% du témoin non contaminé

$2: E_{1}$ : Pourcentage d'épillets présentant des symptômes 25 à $30 \mathrm{j}$ après la contamination

3 : Les résultats suivis de la même lettre ne sont pas différents au seuil 0,05 (ppds de Fischer)

- en cas d'attaque faible comme en 1985 avec la concentration de l'inoculum de $2 \times 10^{5} \mathrm{spores} / \mathrm{ml}$, les variétés peu sensibles Fidel et Talent ne subissent pas de perte de rendement notable.

\section{Évaluation des blés de printemps}

Les résultats présentés ici ne concernent que les notations de symptômes sur épi. Les variétés ayant présenté le meilleur comportement parmi les 147 génotypes étudiés sont présentées dans le tableau III. II s'agit de variétés déjà citées pour leur résistance à $F$ graminearum. Soo mai 3 signalé comme résistant (Yu, 1982) a présenté très peu de symptômes pour 6 années d'étude dans nos essais. Erythrospermum 3086 utilisé comme témoin résistant par Schroeder et Christensen (1963) est un peu plus attaqué.

Cependant, le niveau de résistance de ces variétés est difficile à comparer à celui des meilleurs blés d'hiver dans nos conditions de tests au champ. En effet, en semis d'hiver, elles atteignent le stade floraison parfois très tôt, surtout pour les types peu sensibles à la longueur du jour. En semis de printemps, les dates de floraison sont nettement plus tardives que celles des blés d'hiver et le climat de la période est en général plus sec.

\section{Évaluation des espèces voisines}

Des lignées sensibles à très sensibles ont été trouvées dans toutes les espèces de triticinées étudiées (tableau IV). Des lignées assez résistantes ont été observées surtout chez Triticum monococcum (20\% des lignées testées) et Aegilops speltö̈des $(25 \%)$. Toutefois, les génotypes étudiés pour ces 2 espèces sont beaucoup plus tardifs que les blés tendres d'hiver et la comparaison avec ceux-ci est difficile. Une lignée d'Ae speltoïdes seulement a développé moins de symptômes que les meilleurs blés tendres de printemps. 
Tableau III. Note de sensibilité des blés de printemps ayant présenté le meilleur comportement dans les essais de résistance à la fusariose de l'épi.

\begin{tabular}{|c|c|c|c|}
\hline Nom & $\begin{array}{l}\text { Période } \\
\text { d'étude }\end{array}$ & $\begin{array}{c}\text { Note } \\
\text { moyenne' }\end{array}$ & $\begin{array}{l}\text { Pays } \\
\text { d'origine }\end{array}$ \\
\hline $\begin{array}{l}\text { PI } 94555 \\
\text { PI } 94557 \\
\text { PI } 94558 \\
\text { PI } 94554\end{array}$ & $80-83$ & $\begin{array}{l}2,3 \\
2,7 \\
2,8 \\
2,6\end{array}$ & $\begin{array}{l}\text { Chine } \\
\text { Chine } \\
\text { Chine } \\
\text { Chine }\end{array}$ \\
\hline $\begin{array}{l}7862 \\
\text { Frontana } \\
\text { Toropi } \\
\text { Erythrospermun } \\
7884 \\
\text { Soo mai } 32\end{array}$ & $81-85$ & $\begin{array}{l}2,4 \\
2,9 \\
3,0 \\
3,3 \\
3,2 \\
2,5\end{array}$ & $\begin{array}{l}\text { Chine } \\
\text { Brésil } \\
\text { Brésil } \\
\text { USA } \\
\text { Chine } \\
\text { Chine }\end{array}$ \\
\hline $\begin{array}{l}\text { Ke Han } 6 \\
\text { F } 60049 \\
\text { Long } 75175 \\
\text { Ke Han } 8 \\
\text { You Yi Mai } \\
\text { Rival } \\
\text { Soo mai } 3^{2}\end{array}$ & $83-85$ & $\begin{array}{l}2,1 \\
2,1 \\
2,3 \\
2,7 \\
3,0 \\
3,0 \\
2,3\end{array}$ & $\begin{array}{l}\text { Chine } \\
\text { Chine } \\
\text { Chine } \\
\text { Chine } \\
\text { Chine } \\
\text { USA } \\
\text { Chine }\end{array}$ \\
\hline $\begin{array}{l}\text { Nyu Bay } \\
\text { Nimavi Kyuchu } \\
\text { Soo mai } 3^{2}\end{array}$ & $84-86$ & $\begin{array}{l}2,1 \\
2,2 \\
2,0\end{array}$ & $\begin{array}{l}\text { Japon } \\
\text { Japon } \\
\text { Chine }\end{array}$ \\
\hline $\begin{array}{l}\text { Klein Atlas } \\
\text { LAJ } 2316\end{array}$ & $89-90$ & $\begin{array}{l}2,2 \\
3,1\end{array}$ & $\begin{array}{l}\text { Argentine } \\
\text { Argentine }\end{array}$ \\
\hline
\end{tabular}

1 : Note de 1 (pas de symptômes) à 9 (100\% des épillets atteints).

2: Témoin résistant.

\section{DISCUSSION - CONCLUSION}

Mielke (1988) n'a pas trouvé de résistance intéressante dans une prospection chez 39 espèces de triticinées. Nos résultats montrent que pour 2 espèces, $T$ monococcum et Ae speltoïdes, la proportion de génotypes peu sensibles est plus importante que chez le blé tendre. Dans l'état actuel de nos techniques d'évaluation de la résistance, il n'est pas possible de savoir si les meilleures lignées de ces espèces présentent une résistance supérieure à celle des meilleurs blés tendres.

Les résultats sur les blés de printemps confirment les observations faites par Snijders (1990) qui a trouvé un bon comportement de lignées de blé de printemps citées pour leur résistance à $F$ graminearum, dans les conditions climatiques des Pays-Bas et avec contamination par F culmorum. Ces constatations apportent un élément de plus à l'hypothèse que la résistance à ces 2 champignons pathogènes très voisins est régie par les mêmes mécanismes et que ces géniteurs peuvent être utilisés dans des programmes d'amélioration de la résistance du blé d'hiver à $F$ culmorum.

Même si on ne trouve pas de très haut niveau de résistance, la variabilité pour le comportement vis-à-vis de la fusariose de l'épi est importante chez le blé d'hiver. La fréquence des génotypes résistants est même assez élevée puisque des variétés ayant un bon comportement ont été obtenues sans avoir tenu compte de ce caractère dans le choix des parents, ni avoir réalisé de tests de résistance au cours de la sélection (Festival, Adam, Renan). L'intérêt pratique de ces résistances peut être discuté au regard des résultats obtenus dans des essais réalisés pour mesurer l'influence d'une attaque de Fusarium culmorum sur le rendement : les situations où l'attaque est aussi forte que dans l'essai de 1982 ne se produisent pas en conditions naturelles dans nos régions sauf peut-être pour le blé dur. Des résistances du niveau des meilleures variétés testées devraient être suffisantes pour éviter les pertes de rendement préjudiciables dans une culture. Par contre, les risques de dégâts sont importants pour une variété très sensible même si les conditions ne sont pas très favorables au développement de la maladie.

La contamination de la récolte par des mycotoxines peut se produire même si le niveau d'attaque par le parasite est faible et ne se traduit pas par une baisse de rendement importante (Snijders et Perkowski, 1990). Dans une situation où les exigences de qualité prennent de plus en plus d'importance, des variétés présentant un très haut niveau de résistance seront nécessaires.

L'utilisation de géniteurs d'origines diverses pourrait contribuer, si les systèmes de résistance sont différents, à l'élévation du niveau de résistance chez le blé tendre. La résistance peut être décomposée en au moins 2 facteurs indépendants : résistance à la contamination et résistance à l'extension des symptômes (Schroeder et Christensen, 1963; Saur, 1984). Une connaissance plus précise des géniteurs permettrait de choisir des combinaisons complémentaires pour ces 2 facteurs pour essayer d'obtenir des génotypes transgressifs. Les résistances trouvées dans les autres espèces de triticinées ne sont 
Tableau IV. Comportement de lignées de différentes espèces de triticinées dans les essais de résistance à la fusriose de l'épi.

\begin{tabular}{|c|c|c|c|c|}
\hline Espèce & $\begin{array}{l}\text { Nombre de } \\
\text { lignées } \\
\text { testées }\end{array}$ & $\begin{array}{c}\text { Note1 } \\
\operatorname{maxi}\end{array}$ & $\begin{array}{c}\text { Note }^{2} \\
\text { mini }\end{array}$ & $\begin{array}{c}\text { Nombre de } \\
\text { lignées } \\
\text { de note } \leq 3\end{array}$ \\
\hline$T$ monococcum & 64 & 7,5 & 2,5 & 13 \\
\hline Tpersicum & 49 & 7,5 & 2,5 & 2 \\
\hline Ae squarrosa & 48 & 8,0 & 2,5 & 3 \\
\hline Ae ventricosa & 24 & 7,5 & 5,0 & 0 \\
\hline Ae speltoides & 41 & 7,0 & 1,5 & 11 \\
\hline Ae triuncialis & 33 & 7,5 & 3,0 & 1 \\
\hline Ae cylindrica & 25 & 6,5 & 2,0 & 5 \\
\hline Ae ovata & 19 & 7,5 & 4,0 & 0 \\
\hline Ae triaristata & 14 & 7,5 & 5,0 & 0 \\
\hline Ae biuncialis & 8 & 7,0 & 2,8 & 2 \\
\hline Ae uniaristata & 5 & 7,0 & 5,0 & 0 \\
\hline Ae kotschii & 5 & 7,0 & 5,0 & 0 \\
\hline Ae umbellulata & 4 & 6,0 & 4,5 & 0 \\
\hline Ae variabilis & 4 & 6,5 & 3,0 & 1 \\
\hline Ae comosa & 1 & 3,5 & 3,5 & 0 \\
\hline
\end{tabular}

1 : Note de la lignée la plus sensible de 1 (pas de symptômes) à 9 (100\% des épillets atteints).

2 : Note de la lignée la plus résistance.

pas suffisamment importantes pour justifier leur utilisation en sélection dans un premier temps.

\section{RÉFÉRENCES}

Dickson JG (1942) Scab of wheat and barley and its control. US Dep Agric Farmer's Bull 1599, 1-17

Mesterhazy A (1988) Expression of resistance of wheat to Fusarium graminearum and $F$ culmorum under various experimental conditions. J Phytopathol 123, 304-310

Mielke H (1988) Untersuchungen über Fusarium culmorum (W G Sm) Sacc als Fuss- und Ährenkrankheitserreger beim Weizen. Mitt Biol Bundesanst Land-Forstwirtsch Berl Dahlem 238, $101 \mathrm{p}$

Saur $L$ (1984) Comportement de quatre variétés de blé tendre vis-à-vis de la fusariose de l'épi causée par Fusarium roseum var culmorum (Schwabe) $\mathrm{Sn}$ et $\mathrm{H}$ Agronomie 4, 939-943

Saur L, Benacef N (1991) Relation entre les symptômes de fusariose de l'épi et la perte de rendement chez le blé tendre. Comparaison de quatre variétés. In: Écophysiologie du blé. Les colloques de I'INRA (sous presse)

Schroeder HW, Christensen JJ (1963) Factors affecting resistance of wheat to scab caused by Gibberella zeae (Schw) Petch. Phytopathology 53, 831838

Snijders CHA (1990) Genetic variation for resistance to Fusarium head blight in bread wheat. Euphytica 50, 171-179

Snijders CHA, Perkowski $J(1990)$ Effects of head blight caused by Fusarium culmorum on toxin content and weight of wheat kernels. Phytopathology 80, 566-570

Walther H (1976) Prüfung von Weizen auf Befall mit den Ährenkrankheiten Septoria nodorum und Fusarium culmorum dursch künstliche Infektion im Feldversuch. Tagunsber Akad Landwirtschaftswiss DDR 143, 427-436

Yu YJ (1982) Monosomic analysis for scab resistance and yield components in the wheat cultivar Soo-mo 3. Cereal Res Commun 10, 185-189 